\title{
Herpes simplex virus types 1 and 2 modulate autophagy in SIRC corneal cells
}

\author{
Goran Petrovski ${ }^{1,2}$, Kata Pásztor $^{3}$, László Orosz $^{3}$, Réka Albert ${ }^{1,2}$, Edina Mencel ${ }^{3}$, \\ Morten C Moe ${ }^{4}$, Kai KaArniranta ${ }^{5}$, Andrea Facskó ${ }^{6}$ and Klára Megreri ${ }^{3}$ ** \\ ${ }^{1}$ Department of Ophthalmology, Medical and Health Science Center, \\ University of Debrecen, Debrecen, Hungary \\ ${ }^{2}$ Stem Cells and Eye Research Laboratory and Apoptosis and Genomics Research Group \\ of Hungarian Academy of Sciences, Department of Biochemistry and Molecular Biology, \\ Medical and Health Science Center, University of Debrecen, Debrecen, Hungary \\ ${ }^{3}$ Department of Medical Microbiology and Immunobiology, University of Szeged, \\ Szeged, Hungary \\ ${ }^{4}$ Department of Ophthalmology, Center of Eye Research, Oslo University Hospital, \\ University of Oslo, Oslo, Norway \\ ${ }^{5}$ Department of Ophthalmology, Institute of Clinical Medicine, University of Eastern \\ Finland, and Department of Ophthalmology, Kuopio University Hospital, \\ Kuopio, Finland \\ ${ }^{6}$ Department of Ophthalmology, University of Szeged, Szeged, Hungary \\ *Corresponding author (Fax, +36-62-545-113; Email, megyeri.klara@med.u-szeged.hu)
}

\begin{abstract}
Autophagy and apoptosis function as important early cellular defense mechanisms in infections and other diseases. The outcome of an infection is determined by a complex interplay between the pathogenic microorganism and these intracellular pathways. To better understand the cytopathogenicity of Herpes simplex virus types 1 and 2 (HSV-1 and 2), we studied the effect of these viruses on the autophagic and apoptotic processes in the SIRC corneal cell line. Infection with the KOS strain of HSV-1 and a wild-type strain of HSV-2 enhanced autophagosome formation, triggered cytoplasmic acidification, increased LC3B lipidation and elevated the ratio of apoptotic cells. The autophagy inhibitor bafilomycin A1 triggered a significant increase in the apoptotic responses of HSV-1- and HSV-2-infected cells. Thus, both HSV types affect autophagy and apoptosis in a coordinated fashion, and autophagy plays cytoprotective role in HSV-infected cells via antagonizing apoptosis. Together these data implicate autophagy in the pathogenic mechanism of herpetic keratitis.
\end{abstract}

[Petrovski G, Pásztor K, Orosz L, Albert R, Mencel E, Moe MC, Kaarniranta K, Facskó A and Megyeri K 2014 Herpes simplex virus types 1 and 2 modulate autophagy in SIRC corneal cells. J. Biosci. 39 683-692] DOI 10.1007/s12038-014-9443-y

\section{Introduction}

Herpes simplex virus types 1 and 2 (HSV-1 and -2) belonging to the Herpesviridae family of large, enveloped DNA viruses are capable of causing a wide array of human diseases, including herpes simplex labialis, genital herpes, encephalitis and ocular diseases, such as blepharitis, conjunctivitis, keratitis, iridocyclitis and acute retinal necrosis (Roizman and Pellett 2001; Vemuganti et al. 2011; Babu et al. 2013; Rowe et al. 2013; Silva et al. 2013). During the

Keywords. Apoptosis; autophagy; corneal cells; Herpes simplex virus; keratitis 
course of herpetic keratitis, the HSV-induced cytopathogenicity together with the chronic immune-inflammatory reaction can trigger stromal scarring, thinning and neovascularization, leading to permanent vision impairment. Herpetic keratitis therefore remains a frequent infectious cause of blindness in many countries (Biswas and Rouse 2005; Choudhary et al. 2008; Rowe et al. 2013).

Tissue damage elicited by HSV-1 and -2 displays cell-type-specific features. These viruses cause a lytic infection in the epithelial cells and elicit tissue destruction characterized by complex cell death pattern (Roizman and Pellett 2001; Nguyen and Blaho 2007). In consequence of acquisition, HSV-1 and HSV-2 establish lifelong latency, inhibit apoptosis and maintain cell survival within the neurons (Roizman and Pellett 2001). Systemic and local stressors can interrupt the latency and induce viral reactivation, leading to recrudescent infections. The cytopathogenic mechanism of diseases caused by HSV-1 and HSV-2 involves indirect, immune processes and direct, virus-mediated events (Biswas and Rouse 2005; Holdeman 2005; Kaye and Choudhary 2006; Fatahzadeh and Schwartz 2007; Choudhary et al. 2008).

Multiplication of these viruses subverts the physiological regulation of apoptotic cell demise (Nguyen and Blaho 2007 and 2009). Apoptosis, termed type I programmed cell death, is a type of cell deletion characterized by stereotypic cytomorphological changes, such as nuclear compaction, DNA fragmentation to nucleosomesized fragments and cell shrinkage resulting in cellular breakdown into membrane-bound apoptotic bodies. The molecular events implicated in the activation of apoptotic cascade can be mediated by intrinsic and extrinsic cellular pathways (Nguyen and Blaho 2009). It has been shown that early in infection with HSV-1 or HSV-2, the appearance of viral immediate early (IE) mRNAs activates the cellular apoptotic machinery. However, at 3 to 6 hours post-infection (hpi), several anti-apoptotic HSV proteins [Infected Cell Protein 4 (ICP4), ICP22, ICP17, ICP34.5, US3, US5, US6, UL14 and ICP10 of HSV-2], as well as the latency-associated transcript inhibit apoptotic demise, and maintain cell survival until the replication cycle is completed (Megyeri 2007). The time period while anti-apoptotic HSV proteins are synthesized is termed 'apoptosis prevention window' (Goodkin et al. 2003). In this phase of infection, the intricate balance of pro- and anti-apoptotic factors is strongly biased by the indirect influence of powerful cellular defense pathways, such as the DNA damage response pathway or the interferon system (Shirata et al. 2005). Finally, HSV multiplication leads to the inevitable death of host cells, at least in part via apoptotic mechanisms. Apoptosis fulfils dual functions in the pathogenic mechanism of diseases caused by HSV-1 and HSV-2; it contributes to tissue damage and exerts antiviral effect by inhibiting viral spread at the expense of killing infected cells.

HSV-1 and HSV-2 are also capable of modulating autophagy, termed type II programmed cell death (Tallóczy et al. 2001; Tallóczy et al. 2006; McFarlane et al. 2011; Lussignol et al. 2013). Autophagy is an essential catabolic process that maintains cellular integrity by degrading cytoplasmic constituents and organelles (Deretic and Levine 2009). It is highly activated by several pathological conditions, including nutrient starvation, oxidative stress, inflammation and infections (Deretic and Levine 2009). Macroautophagy (herein referred to as autophagy) is one of the three ways the cells can deliver sequestered cytoplasmic content, damaged proteins, organelles or pathogens to the lysosomal compartment using a double-membraned autophagosomal vacuole as a carrying vehicle (Deretic and Levine 2009). Under conditions of mild stress, autophagy plays a cytoprotective role by eliminating pathogenic microorganisms and dysfunctional organelles. In contrast, under conditions leading to massive cell damage, excessive autophagy possesses destructive power culminating in cell demise (Chen and Klionsky 2011). It has been shown that at the early stage of infection HSV-1 provokes autophagy (McFarlane et al. 2011). However, some viral proteins (ICP34.5 and US11) synthesized during the subsequent phases of productive infection function as powerful inhibitors of the autophagic process (Tallóczy et al. 2001; Tallóczy et al. 2006; Orvedahl et al. 2007; Lussignol et al. 2013). ICP34.5 of HSV-1 antagonizes autophagy by binding Beclin-1, and by blocking the double-stranded RNA-dependent protein kinase/eukaryotic translation initiation factor $2 \alpha$ (PKR/ eIF2 $\alpha$ ) pathway (Tallóczy et al. 2006; Orvedahl et al. 2007). The HSV-1 US11 protein exerts anti-autophagic activity via its direct interaction with PKR (Lussignol et al. 2013). These interesting studies have shed light on the importance of autophagy in infections caused by HSV-1, and have successfully uncovered the underlying molecular mechanisms in some experimental systems. At present, much less is known about the effects of wildtype HSV-2 on autophagy.

Our group previously described the importance of apoptosis in the cytopathogenicity of HSV-1 and demonstrated the role of the pro-apoptotic p53 family member TAp63 $\gamma$ in the pathogenic mechanism of ocular surface disease caused by HSV-1 (Orosz et al. 2010). To further understand the corneal processes induced by HSV-1 and HSV-2, in the present study we investigated the effects of these viruses on autophagy in the Statens Seruminstitut Rabbit Cornea (SIRC) cell line, which displays many features of corneal keratocytes (Niederkorn et al. 1990). 


\section{Materials and methods}

\subsection{Cell culture and HSV growth}

The SIRC cell line, was grown in Dulbecco's modified Eagle's minimal essential medium (Sigma Chemical Co., St. Louis, MO, USA) supplemented with $10 \%$ fetal calf serum (FCS) at $37^{\circ} \mathrm{C}$ in a $5 \% \mathrm{CO}_{2}$ atmosphere. For experiments, SIRC cell cultures were inoculated with the KOS strain of HSV-1 or a wild-type strain of HSV-2 at different multiplicities of infection (MOIs). Virus plaque assays were performed on confluent monolayers of Vero cells inoculated with one or other HSV solution for $1 \mathrm{~h}$ at $37^{\circ} \mathrm{C}$ and overlaid with $0.5 \%$ agarose in phenol red-free Eagle's minimum essential medium supplemented with $7.5 \%$ FCS and $2 \mathrm{mM}$ L-glutamine. Plaque titers were determined at 3 days after HSV infection.

\subsection{Indirect immunofluorescence assay}

Cytospin cell preparations were fixed in methanol-acetone (1:1) for $15 \mathrm{~min}$ at $-20^{\circ} \mathrm{C}$. The slides were incubated with specific antibodies to HSV glycoprotein D [(gD) (Sigma)], autophagy-related gene 5 (Atg5) or microtubule-associated protein 1 light chain $3 \mathrm{~B}(\mathrm{LC} 3 \mathrm{~B})$ for $1 \mathrm{~h}$ at $37^{\circ} \mathrm{C}$. After washing with phosphate-buffered saline (PBS), the samples were reacted with fluorescein isothiocyanate (FITC)-conjugated species-specific antibodies (1:160) (Sigma) and incubated for $1 \mathrm{~h}$ at $37^{\circ} \mathrm{C}$. After washing with PBS, the cells were visualized by epifluorescence microscopy (Leica Microsystems GmbH, Wetzlar, Germany). The ratio of positive to negative cells was determined after counting 1,000 cells in random fields.

\subsection{Transmission electron microscopy (TEM)}

Samples were fixed in $0.1 \mathrm{M}$ sodium cacodylate-buffered $2.5 \%$ glutaraldehyde solution $(\mathrm{pH} 7.4)$ for $2 \mathrm{~h}$ and then rinsed three times for 10 min each in $0.1 \mathrm{M}$ sodium cacodylate buffer and $7.5 \%$ saccharose. The samples were post-fixed in $1 \%$ $\mathrm{OsO}_{4}$ solution for $1 \mathrm{~h}$. After dehydration in an ethanol gradient (70\%, 96\% and $100 \%$ ethanol for 20 min each), samples were embedded in Durcupan ACM. Ultrathin sections were stained with uranyl acetate and lead citrate. Sections were examined in a Philips CM 10 microscope (Philips Electronic Instruments, Mahwah, NJ, USA) at $80 \mathrm{kV}$.

\subsection{Acridine orange staining}

Autophagy was assessed by the acridine orange staining procedure of autophagic vacuoles. The cells, grown on glass coverslips were incubated for $15 \mathrm{~min}$ at $37^{\circ} \mathrm{C}$ in serum-free medium containing $2 \mu \mathrm{g} / \mathrm{mL} \mathrm{3,6-bis(dimethylamine)acridine.}$ After cells had been washed, fluorescent micrographs were obtained using an epifluorescence microscope (Leica Microsystems $\mathrm{GmbH}$ ). Acridine orange accumulated by acidic compartments emits orange fluorescence, whereas this metachromatic dye bound to compartments with slightly acidic or neutral $\mathrm{pH}$ emits green fluorescence.

\subsection{Quantification of apoptosis by annexin V/propidium iodide (AN/PI) double labelling}

For AN/PI double labelling, the cells were stained with FITClabelled annexin V (AN-FITC) and propidium iodide (PI) (MBL, Woburn, MA, USA) according to the manufacturer's instructions. The fluorescence intensities of AN-FITC and PI were determined with a BD Bioscience flow cytometer (BD Biosciences, San Diego, CA, USA). The percentages of apoptotic cells were calculated by sorting the cells that were positive only for AN (early apoptotic stage) or for both AN and PI (late apoptotic or secondary necrotic stages).

\subsection{Quantification of apoptosis by enzyme-linked immunosorbent assay (ELISA)}

For apoptosis ELISA, the cells were processed in a cell death detection ELISA kit (Roche Diagnostics GmbH, Penzberg, Germany). The cells were incubated in lysis buffer for $30 \mathrm{~min}$ and centrifuged at $8000 \mathrm{rpm}$ for $10 \mathrm{~min}$. The supernatants were transferred into a streptavidin-coated microplate and incubated with biotin-conjugated anti-histone and peroxidase-conjugated anti-DNA monoclonal antibodies for $2 \mathrm{~h}$. After washing, ABTS substrate solution was added to each well for $15 \mathrm{~min}$. Absorbance was measured at 405 and $490 \mathrm{~nm}$. The specific enrichment of mono- and oligonucleosomes was calculated as

enrichment factor $(\mathrm{EF})=$ absorbance of HSV-infected cells/absorbance of corresponding non-infected control cells

\subsection{Western blot assays}

Cells were homogenized in CytoBuster lysis buffer (Novagen, EMD Biosciences, Darmstadt, Germany), and the mixture was then centrifuged at $10,000 \mathrm{~g}$ for $10 \mathrm{~min}$ to remove cell debris. Protein concentrations of cell lysates were determined by using the Bio-Rad protein assay (BioRad, Hercules, CA, USA). Supernatants were mixed with 
Laemmli's sample buffer and boiled for $3 \mathrm{~min}$. Aliquots of the supernatants, containing $50 \mu \mathrm{g}$ of total protein to detect LC3B were resolved by SDS-PAGE and electrotransferred onto nitrocellulose filters (Bio-Rad). Preblocked blots were reacted with polyclonal antibody against LC3B (Sigma) for $4 \mathrm{~h}$ in PBS containing $0.05 \%$ Tween $20,1 \%$ dried non-fat milk (Difco Laboratories, Detroit, MI, USA) and 1\% BSA (Sigma). Blots were then incubated for $2 \mathrm{~h}$ with peroxidase-conjugated anti-rabbit antibody (DakoCytomation, Carpinteria, CA, USA). Filters were developed by using a chemiluminescence detection system (Thermo Fisher Scientific Inc., Rockford, IL, USA). The autoradiographs were scanned with a GS-800 densitometer (Bio-Rad), and the relative band intensities were quantified by use of the ImageQuant software (Molecular Dynamics, Sunnyvale, CA, USA).

\subsection{Statistical analysis}

All values are expressed as mean \pm standard deviation (SD). The one-way ANOVA test with the Bonferroni post-test was used for pairwise multiple comparisons, and $p$-values $<0.05$ were considered statistically significant (GraphPad Prism 5, GraphPad Software Inc., La Jolla, CA, USA).

\section{Results}

\subsection{Effects of HSV-1 and HSV-2 on autophagy in the SIRC cell line}

To investigate the susceptibility of SIRC cells to the KOS strain of HSV-1 and a wild-type HSV-2 strain, virus multiplication was assessed by using indirect immunofluorescence assay, Western blot and plaque titration. The data demonstrated that both HSV-1 and HSV-2 could establish a productive infection in this cell line (data not shown; Orosz et al. 2010).

To study the effects of HSV-1 and HSV-2 on the process of autophagy, SIRC cells were infected either with HSV-1 or HSV-2 at an MOI of 1 and analysed by TEM and immunofluorescence assay at $24 \mathrm{hpi}$.

TEM examination of the mock-infected culture revealed normal cell morphology (figure 1A). In contrast, there were numerous intracytoplasmic vacuoles in HSV-1-infected cells (figures 1B-D). These vacuolar structures contained amorphous materials, organelles or virion particles (figures 1BD). The vacuoles were dispersed in the cytoplasm evenly (figures 1B-D). This finding corresponds to the TEM picture of excessive autophagy. This test has also provided experimental proof of ongoing viral replication in SIRC cells, as numerous assembled capsids and primarily enveloped virions were revealed in the nuclei of infected cells. Some fully matured cytoplasmic capsids and virions released from the cell in exocytotic vesicles were also detected (figures 1B-D).

Indirect immunofluorescence assay to investigate the effect of HSV-1 on the intracellular localization of Atg5 and LC3B revealed diffuse, cytoplasmic staining patterns in the mock-infected cultures (figures $1 \mathrm{E}$ and $\mathrm{G}$, respectively). In contrast, HSV-1 increased the intensity of staining and triggered translocation of Atg5 and LC3B from the cytoplasm to distinct puncta in $67 \%$ and $93 \%$ of cells, respectively (figures $1 \mathrm{~F}$ and $\mathrm{H}$, respectively). Together, these data demonstrate that HSV-1 triggers the formation of autophagic vacuoles, and alters the intracellular localization of Atg5 and LC3B.

To investigate the autophagic flux, bafilomycin A1 (BFLA) was used as a pharmacological tool to inhibit lysosomal degradative activity and to prevent fusion of autophagosomes with lysosomes. As BFLA decreased the production of infectious virions severely when it was present throughout the infection (data not shown), an experimental strategy that allows adsorption and uncoating to proceed undisturbed was applied (figure 2A) (Harley et al. 2001). The cells were infected either with HSV-1 or HSV-2 at an MOI of 20, and incubated for $3 \mathrm{~h}$. The cultures were then treated with BFLA for an additional $12 \mathrm{~h}$ period, and analysed for viral yields, apoptosis and LC3B expression (figure 2A). Western blot analysis to quantify intracellular $\mathrm{gD}$ protein revealed two bands migrating near 52 and $56 \mathrm{kDa}$ in HSV-1- or HSV-2-infected cells (figure 2B). This result is fully consistent with previous observations demonstrating that gD migrates as a doublet; the $\sim 52$ and $\sim 56 \mathrm{kDa}$ polypeptides correspond to the nonglycosylated and fully glycosylated forms of $\mathrm{gD}$, respectively (Balachandran et al. 1982; Isaacs et al. 2000; Gianni et al. 2009). BFLA inhibited the accumulation of this envelope glycoprotein in HSV-1- or HSV-2-infected cells by $12.3 \%$ and $42.5 \%$, respectively (figure 2B). Plaque assay to quantify viral multiplication revealed that BFLA retarded replication of HSV-1 and HSV-2 by $52.6 \%$ and $13.6 \%$, respectively (figure 2C). Thus, the strategy involving delayed addition of BFLA provided conditions when productive infection has occurred, though at diminished levels compared to infected cultures incubated in the absence of this autophagy inhibitor. Western blot analysis to measure the levels of the lipidated and nonlipidated forms of LC3B demonstrated that the mockinfected culture displayed endogenous expression both of LC3B-II and LC3B-I; the LC3B-II / LC3B-I ratio was 0.17 at $15 \mathrm{~h}$ of culturing (figure 2D, lane 6). Cells infected with HSV-1 and HSV-2 displayed elevated LC3B-II and depressed LC3B-I levels; the LC3B-II / LC3B-I ratios were 0.82 and 0.56 , respectively (figure $2 \mathrm{D}$, lanes 2 and 4 , respectively). This result demonstrates that HSV-1 and HSV-2 promote LC3B lipidation. BFLA highly elevated the level of 

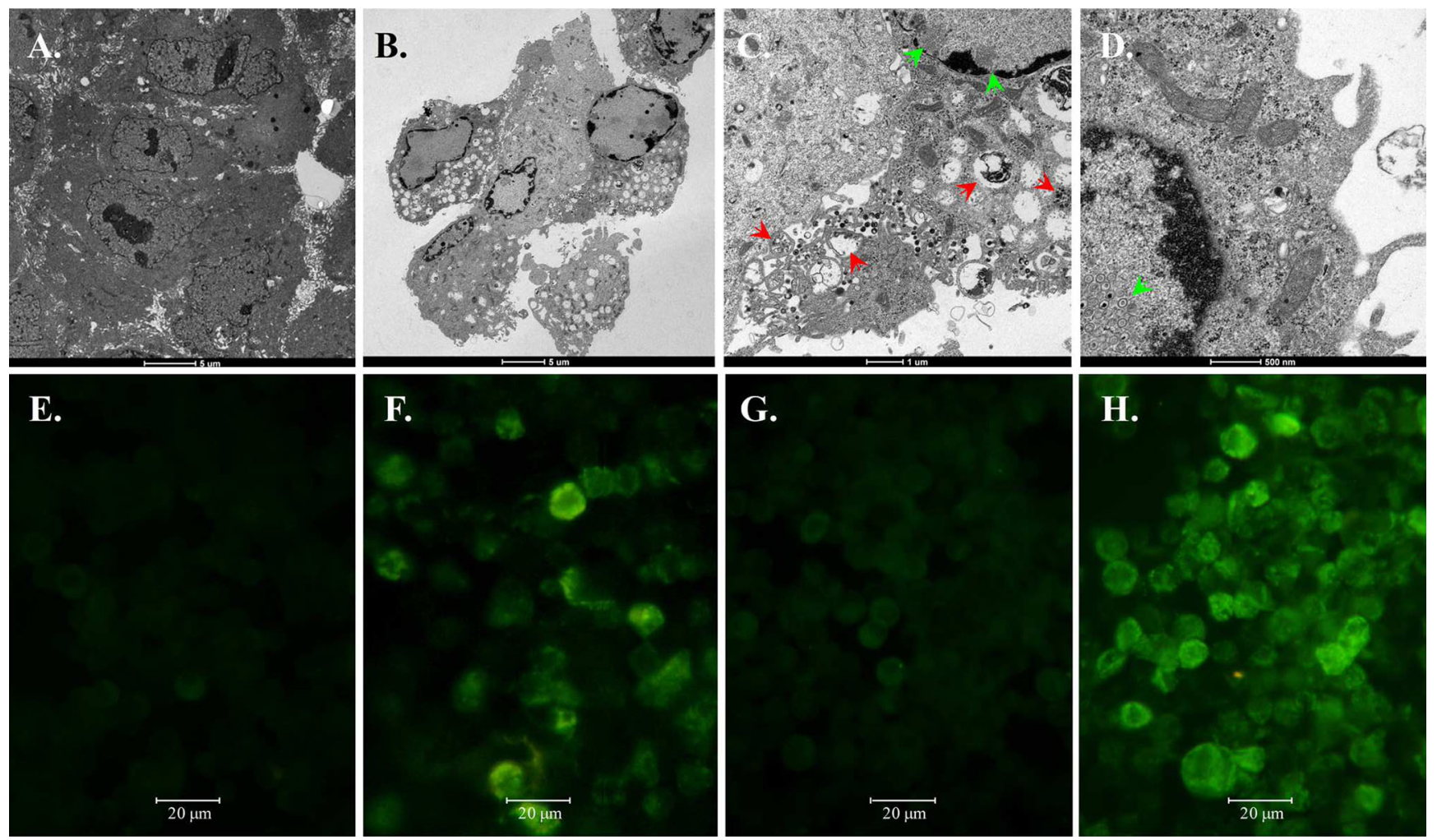

Figure 1. HSV-1 infection increases the formation of autophagic vacuoles in SIRC cells. (A-D) Formation of autophagic vacuoles as assessed by TEM: (A) Mock-infected cells; (B, C and D) cells infected with HSV-1. Red and green arrows indicate autophagic vacuoles and viral particles, respectively. (E and $\mathbf{F}$ ) Immunofluorescence assay for localization of Atg5: (E) Mock-infected cells; (F) cells infected with HSV-1. (G and $\mathbf{H})$ Immunofluorescence assay for localization of LC3B: (G) Mock-infected cells; (H) cells infected with HSV-1.

LC3B-II (figure 2D, lane 1), indicating that this drug efficiently blocked autophagy under the experimental conditions used, and the pathways promoting LC3B-II accumulation are intact in the SIRC cell line. In the presence of BFLA, HSV-1 and HSV-2 triggered slightly lower increase in the level of LC3B-II than that observed in the corresponding drug control (figure 2D, lanes 3, 5 and 1, respectively). Thus, both HSV-1 and HSV-2 decrease the autophagic flux to some extent.

Acridine orange staining to detect autophagic vacuoles revealed green cytoplasmic and nuclear staining in the mock-infected cells at $24 \mathrm{~h}$ of culturing (figure $3 \mathrm{~A}$ ). The nuclei of mock-infected cells were mostly round in shape (figure $3 \mathrm{~A}$ ). In contrast, cells infected with HSV-1 or HSV-2 at an MOI of 1 displayed green nuclear and strong red cytoplasmic fluorescence at $24 \mathrm{hpi}$, demonstrating that both HSV types promote cytoplasmic acidification (figure $3 \mathrm{~A}$ ). In HSV-1-infected cultures a few small syncytia, containing $<5$ nuclei, were observed, while HSV-2 infection resulted in the formation of numerous large multinucleated giant cells, which contained $>50$ nuclei (figure $3 \mathrm{~A}$ ). The nuclei of infected cells had markedly irregular shape, contained disorganized chromatin structures, and in some areas blurring, folding or complete disruption of the nuclear membrane was also observed (figure $3 \mathrm{~A}$ ).

\subsection{Cell death pattern of HSV-1- and HSV-2-infected SIRC cells}

To investigate the cytopathogenicity of HSV-1 and HSV-2 in SIRC cultures, the cell death pattern of cultures infected at an MOI of 1 was determined by AN/PI double labelling at 24 hpi.

Both HSV-1 and HSV-2 decreased the proportions of viable cells by $32.9 \%$ and $57.8 \%$, respectively (figure $3 \mathrm{~B}$ ). The proportions of AN-single-positive (early apoptotic) and double-positive (late apoptotic or secondary necrotic) cells in 
A infection BFLA

$\downarrow 3 \mathrm{~h} \downarrow \quad 12 \mathrm{~h}$

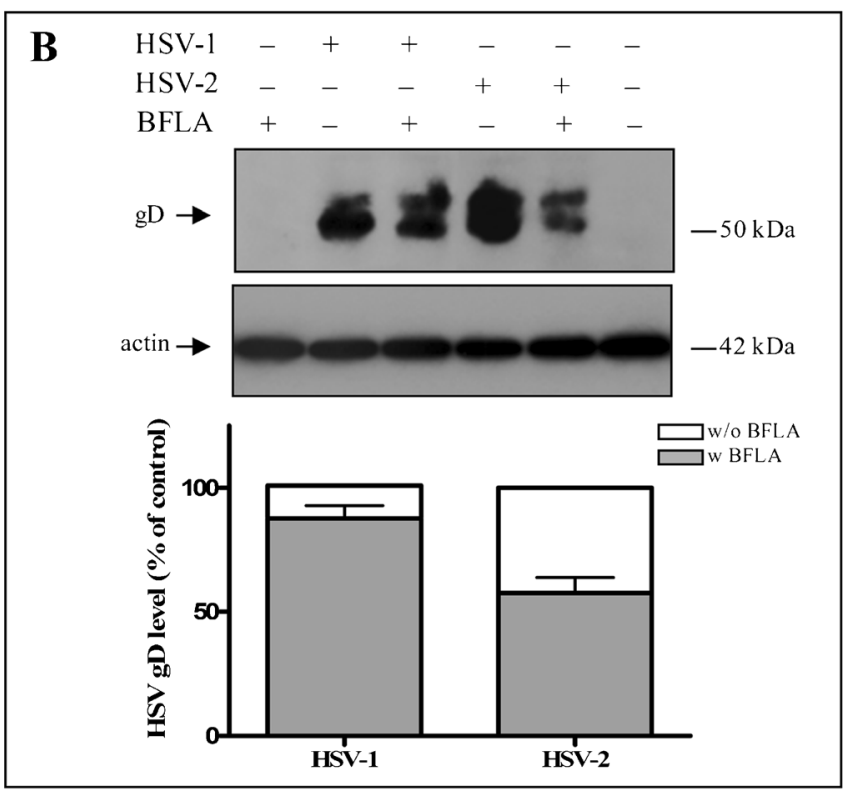

C
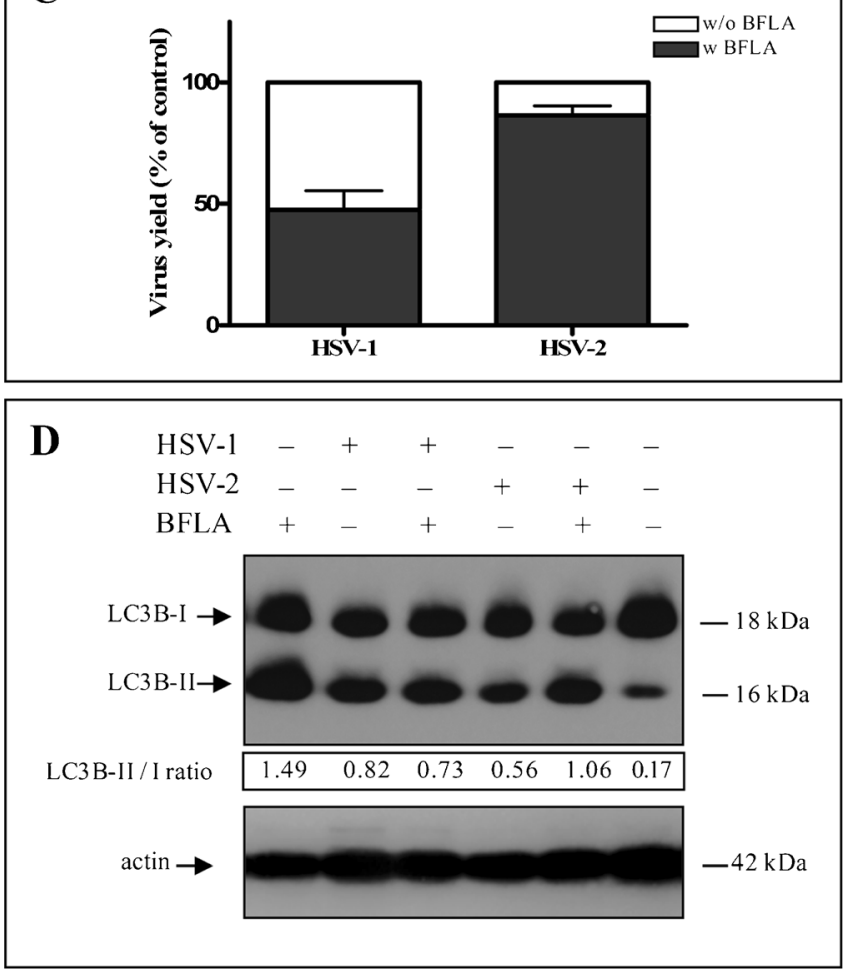

HSV-1-infected cultures were increased by $15.4 \%$ and $13.3 \%$, respectively (figure $3 \mathrm{~B}$ ). The proportions of double-positive and PI-single positive (necrotic) cells were increased by $36.5 \%$ and $23.8 \%$ in HSV-2-infected cultures,
4 Figure 2. HSV-1 and HSV-2 trigger LC3B lipidation, without increasing the autophagic flux. (A) Graphical scheme of the experiments designed to assess autophagic flux. (B) Detection of HSV gD and actin by Western blot assay. Relative densities of HSV gD and actin were measured by densitometric analysis of western blots. HSV gD was normalized to actin, percentage changes of gD levels in HSV-infected cells incubated with BFLA were compared to infected controls incubated in the absence of BFLA, and the results are presented in graph form below the image. (C) Detection of viral yield by plaque assay. (D) Detection of LC3B and actin by Western blot assay. Relative densities of LC3B-I, LC3B-II and actin were measured by densitometric analysis of western blots. LC3B-I and LC3B-II were normalized to actin, and the LC3B-II to LC3B-I ratio is shown below each lane.

respectively (figure $3 \mathrm{~B}$ ). Together, these data reveal that both HSV-1 and HSV-2 trigger extensive cell demise.

\subsection{Inhibition of autophagy increases apoptotic rates of SIRC cells infected with $\mathrm{HSV}-1$ and $\mathrm{HSV}-2$}

To investigate the potential connection between autophagy and apoptosis, the effect of the autophagy inhibitor BFLA on the extent of apoptosis was assessed. The cells were infected either with HSV-1 or HSV-2 at an MOI of 20, and incubated for $3 \mathrm{~h}$. The cultures were then treated with BFLA for an additional $12 \mathrm{~h}$ period, and analysed for apoptosis by ELISA. The assay revealed significantly higher apoptotic rates in HSV-1- and HSV-2-infected cells; the EFs for HSV-1 and HSV-2 were 2.86 and 2.93, respectively (figure 4). EFs for the HSV-1- and HSV-2-infected cells incubated in the presence of BFLA were 6.36 and $4.32 \mathrm{vs}$ 2.86 and 2.93 for the HSV-1- and HSV-2-infected cells incubated in the absence of drug, respectively (figure 4). Thus, BFLA triggered a significant increase in the apoptotic responses of HSV-1- and HSV-2-infected cells as compared with the corresponding infected controls incubated in the absence of drug. Moreover, there was a significant difference between the apoptosis inducing activities of HSV-1 and HSV-2 in BFLA-treated cells (figure 4). Together, these results demonstrate that inhibition of autophagy facilitates apoptosis induced by HSV-1 and HSV-2.

\section{Discussion}

Apoptosis and autophagy are implicated in the maintenance of ocular surface integrity both in physiological and pathological conditions (Wilson 1999; Vemuganti et al. 2004; Buron et al. 2006; Zoukhri et al. 2008). Compelling evidence indicates that apoptosis is instrumental in glaucoma, retinitis pigmentosa, cataract, retinoblastoma, retinal ischemia, diabetic retinopathy and several eye infections caused 

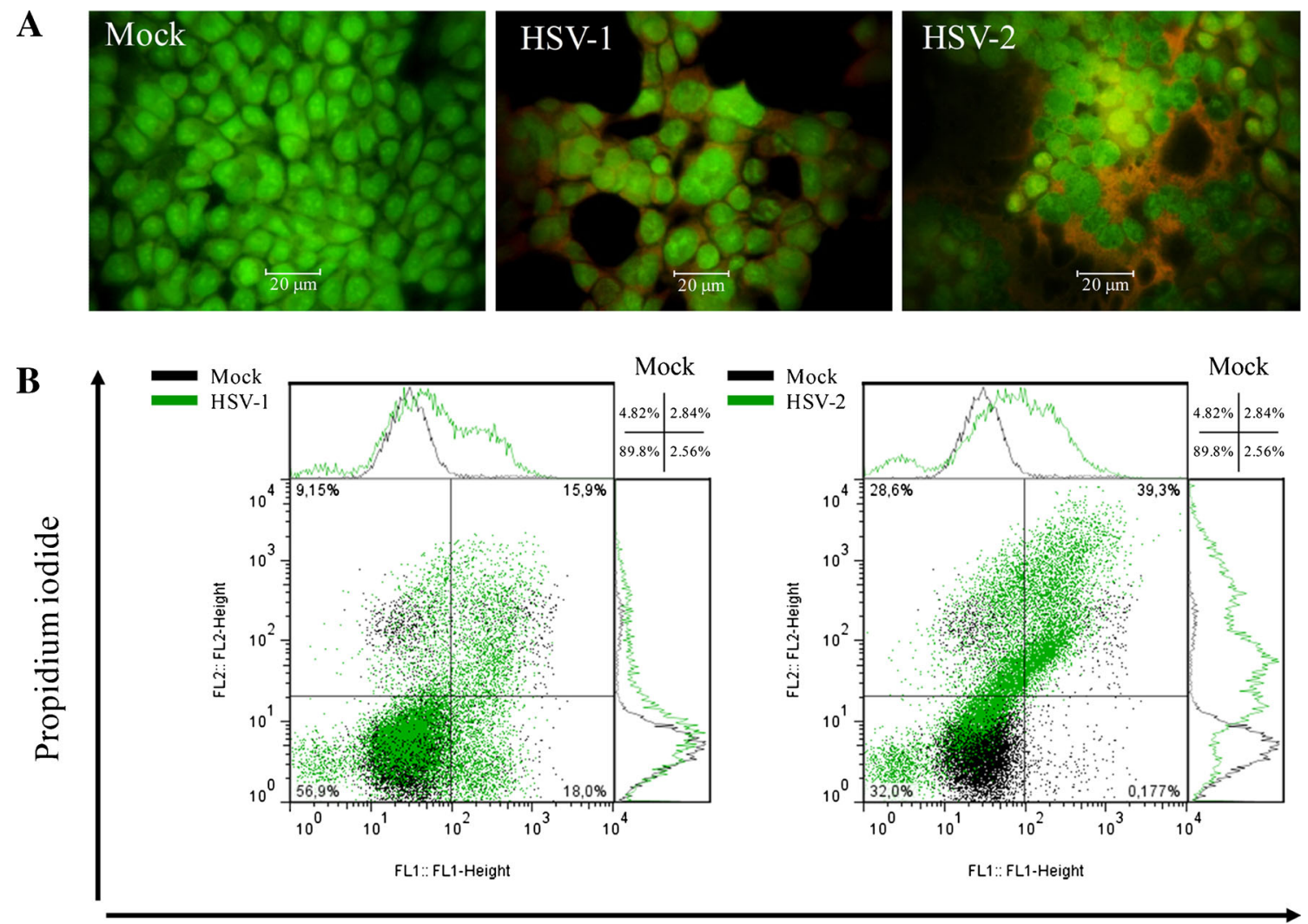

Annexin V (FITC)

Figure 3. HSV-1 and HSV-2 trigger cytoplasmic acidification and excessive cell death in SIRC cells. (A) Intracellular pH as assessed by acridine orange staining. (B) Cell death pattern as assessed by AN/PI double labelling. The fluorescence intensities of AN-FITC and PI were determined by flow cytometry. The two graphs show superimposed dot plots of fluorescence intensities either of HSV-1 or HSV-2 and mock-infected cells.

by viruses, bacteria, fungi and some protozoans (Kaneda et al. 1999; Vemuganti et al. 2004; Cottet and Schorderet 2009; Zhang et al. 2010; Poznic M 2009; Barber et al. 2011; Pinazo-Durán et al. 2013). In contrast, we are just beginning to understand the role of autophagy in ocular diseases. As herpetic keratitis continues to be a frequent and serious medical problem, in this study we have investigated the role of apoptosis and autophagy in the pathogenic mechanism of direct corneal damage triggered by HSV-1 and HSV-2 in the SIRC corneal cell line.

Remarkable studies focusing on the function of corneal keratocytes in the maintenance of visual integrity have clearly demonstrated that keratocytes, located in the corneal stroma as a highly interconnected cellular network, undergo apoptotic cell demise immediately after infections (Vemuganti et al. 2004; West-Mays et al. 2006). The apoptotic response of keratocytes operates as an important antiviral mechanism by inhibiting the spread of viruses to deeper structures of the eye, and minimizes the inflammation that accompanies corneal injury (Wilson et al. 1997; Vemuganti et al. 2004; Morishige et al. 2006; Ambrósio et al. 2009). In full accord with these findings our data have corroborated that both HSV-1 and HSV-2 trigger apoptosis in corneal cells.

Further interesting studies have demonstrated that the effect of HSV-1 on autophagy is cell-type-specific. While HSV-1 activates the STING (stimulator of interferon genes)dependent autophagy pathway in murine macrophages and dendritic cells, neuroblastoma cells respond to HSV-1 infection with an abortive autophagy, and the anti-autophagic effects of ICP34.5 and Us11 proteins dominate in fibroblasts and primary murine neurons (Tallóczy et al. 2001; Tallóczy et al. 2006; Orvedahl et al. 2007; Santana et al. 2012; Lussignol et al. 2013). However, the impact of HSV infection on the autophagic process has not yet been elucidated in corneal cells. Our analyses have revealed that HSV-1 facilitates the accumulation of autophagosomes, as well as the recruitment of LC3B and Atg5 to autophagic vacuoles in the 


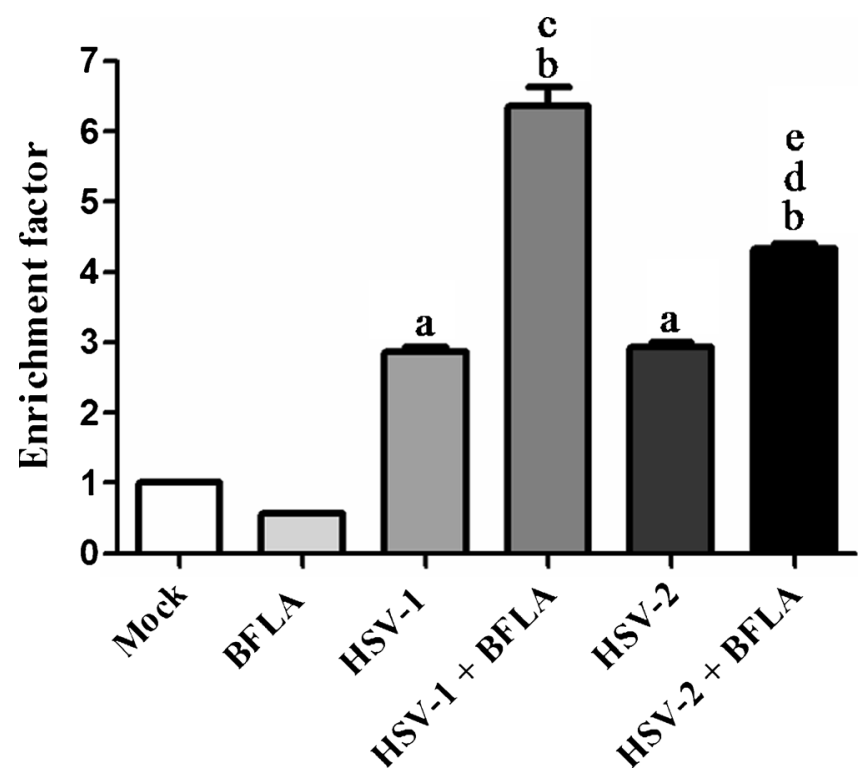

Figure 4. Bafilomycin A1 decreases apoptosis in HSV-1- and HSV-2-infected cells. Apoptosis was detected by ELISA. The enrichment factor was calculated as described in the Material and Methods. Data are mean $( \pm \mathrm{SD})$ values from three independent experiments. $p$-Values were calculated by the ANOVA test with the Bonferroni post-test. ${ }^{\mathrm{a}} p<0.001$ vs Mock; ${ }^{\mathrm{b}} p<0.001$ vs BFLA; ${ }^{\mathrm{c}} p<0.001$ vs HSV-1; ${ }^{\mathrm{d}} p<0.001$ vs HSV-2; ${ }^{\mathrm{e}} p<0.001$ vs HSV$1+$ BFLA.

SIRC cell line. Our further experiments have also shown that both HSV-1 and HSV-2 trigger LC3B lipidation, and increase cytoplasmic acidification, without enhancing the autophagic flux. Together, these results demonstrate that autophagy is induced by both HSV-1 and HSV-2 in spite of their effective anti-autophagic armamentarium, which possibly impedes the process considerably via inhibiting the fusion of autophagosomes with lysosomes.

Recent findings demonstrated that the apoptotic, autophagic and necrotic pathways are interconnected and subject to coordinated regulation (Jain et al. 2013). Autophagy can operate as antagonist, facilitator or cooperator of apoptosis depending on the nature of stressor and cellular context (Jain et al. 2013). It has also been shown that HSV-1 elicits an mTOR-independent pathway of autophagy, which in turn prolongs survival of U251 glioma cells by counteracting the coinciding apoptotic response (Tovilovic et al. 2013). Thus, next we have asked whether autophagy has the potential to modulate apoptotic demise during the course of HSV1 and HSV-2 infection. Our results have revealed that apoptotic cell demise induced by both HSV-1 and HSV-2 is markedly enhanced by inhibition of autophagy indicating that autophagy acts as antagonist of apoptosis in HSVinfected SIRC cells. Induction of autophagy by HSV-1 and
HSV-2 may represent a viral strategy to provide sufficient time for replication via inhibiting untimely apoptotic demise. These data corroborate previous findings obtained with HSV-1-infected U251 glioma cultures (Tovilovic et al. 2013), and extend the spectrum of cells in which HSVmediated induction of autophagy alleviates apoptosis. Under circumstances when apoptosis is blocked, viral replication can proceed in an undisturbed fashion, and the inevitable demise of HSV-infected cells may preferably occur by way of necrosis. This type of cell death may trigger a strong proinflammatory response and thereby it may contribute to the development of characteristic clinical symptoms seen in keratitis patients. Together, these results may bear on keratitis, since dysregulation of keratocyte apoptosis may account for the structural and functional damage of cornea occurring during the course of HSV infection. Moreover, our further observation demonstrating a more remarkable anti-apoptotic effect of autophagy in cultures infected with HSV-1 than in HSV-2-infected cells suggests that the mechanism of corneal cytopathogenicity evoked by the two HSV types might involve some different molecular mechanisms.

A better understanding of intrinsic autophagy evolving during the course of herpes simplex keratitis and other infections caused HSV-1 and HSV-2 may provide useful knowledge for the development of novel therapeutic modalities acting through autophagy modulation. A recent interesting study revealed that rapamycin used topically in a mouse model of HSK suppressed inflammation and neovascularization (Zapata et al. 2012). In view of our present findings obtained by using an in vitro SIRC corneal cell model system, it is reasonable to infer that the beneficial therapeutic efficiency of autophagy inducers in herpetic keratitis may partly be due to their ability to reduce tissue damage via counteracting apoptotic demise of infected cells.

Overall, this study demonstrates that HSV-1 and HSV-2 affect autophagy and apoptosis in a coordinated fashion, and autophagy antagonizes apoptosis in HSV-infected cells. Together these data implicate autophagy in the pathogenic mechanisms of herpetic keratitis.

\section{Acknowledgements}

We would like to thank Sigurd Boye, Gyöngyi Ábrahám and Erika Berényi for expert technical assistance and support. This work was supported by the TÁMOP-4.2.2/B-10/12010-0012, TÁMOP4.2.2.A-11/1/KONV-2012-0035 programs of the Hungarian National Development Agency, the Hungarian Scientific Research Fund (OTKA PD 101316), the EVO grants of Kuopio University Hospital, the Finnish Cultural Foundation and its North Savo Fund, the Finnish Eye Foundation, the Finnish Funding Agency for Technology and Innovation, the Health Research Council of the Academy of Finland, the Päivikki and Sakari Sohlberg 
Foundation, the Research Council of Norway, the Norwegian Center for Stem Cell Research, the Norwegian Association of the Blind and Partially Sighted, Blindemisjonen IL, the Faculty of Medicine University of Oslo and Oslo University Hospital.

\section{References}

Ambrósio R Jr, Kara-José N and Wilson SE 2009 Early keratocyte apoptosis after epithelial scrape injury in the human cornea. Exp. Eye Res. 89 597-599

Babu K, Adiga M, Govekar SR, Kumar BR and Murthy KR 2013 Associations of Fuchs heterochromic iridocyclitis in a South Indian patient population. J. Ophthalmic. Inflamm. Infect. 1514

Balachandran N, Harnish D, Rawls WE and Bacchetti S 1982 Glycoproteins of herpes simplex virus type 2 as defined by monoclonal antibodies. J. Virol. 44 344-355

Barber AJ, Gardner TW and Abcouwer SF 2011 The significance of vascular and neural apoptosis to the pathology of diabetic retinopathy. Invest. Ophthalmol. Vis. Sci. 52 1156-1163

Biswas PS and Rouse BT 2005 Early events in HSV keratitissetting the stage for a blinding disease. Microbes Infect. 7 799-810

Buron N, Micheau O, Cathelin S, Lafontaine PO, Creuzot-Garcher C and Solary E 2006 Differential mechanisms of conjunctival cell death induction by ultraviolet irradiation and benzalkonium chloride. Invest. Ophthalmol. Vis. Sci. 47 4221-4230

Chen Y and Klionsky DJ 2011 The regulation of autophagy unanswered questions. J. Cell Sci. 124 161-170

Choudhary A, Higgins G and Kaye SB 2008 Herpes simplex keratitis and related syndromes; in Cornea and external eye disease (eds) GK Krieglstein and RN Weinreb (Heidelberg: Springer) pp 123-144

Cottet S and Schorderet DF 2009 Mechanisms of apoptosis in retinitis pigmentosa. Curr. Mol. Med. 9 375-383

Deretic V and Levine B 2009 Autophagy, immunity, and microbial adaptations. Cell Host Microbe 5 527-549

Fatahzadeh M and Schwartz R 2007 Human herpes simplex virus infections: Epidemiology, pathogenesis, symptomatology, diagnosis, and management. J. Am. Acad. Dermatol. 57 737-763

Gianni T, Amasio M and Campadelli-Fiume G 2009 Herpes simplex virus $\mathrm{gD}$ forms distinct complexes with fusion executors $\mathrm{gB}$ and $\mathrm{gH} / \mathrm{gL}$ in part through the C-terminal profusion domain. $J$. Biol. Chem. 284 17370-17382

Goodkin ML, Ting AT and Blaho JA 2003 NF-kappaB is required for apoptosis prevention during herpes simplex virus type 1 infection. J. Virol. 77 7261-7280

Harley CA, Dasgupta A and Wilson DW 2001 Characterization of herpes simplex virus-containing organelles by subcellular fractionation: role for organelle acidification in assembly of infectious particles. J. Virol. 75 1236-1251

Holdeman NR 2005 Herpes simplex virus: ocular manifestations; in Ocular therapeutics handbook: A clinical manual 2nd edition (eds) BE Onofrey, L Skorin and NR Holdeman (Philadelphia: Lippincott Williams \& Wilkins) pp 208-210
Isaacs CE, Xu W, Pullarkat RK and Kascsak R 2000 Retinoic acid reduces the yield of herpes simplex virus in Vero cells and alters the N-glycosylation of viral envelope proteins. Antiviral Res. 47 $29-40$

Jain MV, Paczulla AM, Klonisch T, Dimgba FN, Rao SB, Roberg K, Schweizer F, Lengerke C, et al. 2013 Interconnections between apoptotic, autophagic and necrotic pathways: implications for cancer therapy development. J. Cell. Mol. Med. 17 12-29

Kaneda K, Kashii S, Kurosawa T, Kaneko S, Akaike A, Honda Y, Minami M and Satoh M 1999 Apoptotic DNA fragmentation and upregulation of Bax induced by transient ischemia of the rat retina. Brain Res. 815 11-20

Kaye S and Choudhary A 2006 Herpes simplex keratitis. Prog. Retin. Eye Res. 25 355-380

Lussignol M, Queval C, Bernet-Camard MF, Cotte-Laffitte J, Beau I, Codogno P and Esclatine A 2013 The Herpes simplex sirus 1 Us11 protein inhibits autophagy through its interaction with the protein kinase PKR. J. Virol. 87 859-871

McFarlane S, Aitken J, Sutherland JS, Nicholl MJ, Preston VG and Preston CM 2011 Early induction of autophagy in human fibroblasts after infection with human cytomegalovirus or herpes simplex virus 1. J. Virol. 85 4212-4221

Megyeri K 2007 Modulation of apoptotic pathways by herpes simplex viruses; in Latency strategies of herpesviruses (eds) J Minarovits, E Gonczol and T Valyi-Nagy (New York: Springer) pp 37-54

Morishige N, Jester JV, Naito J, Osorio N, Wahlert A, Jones C, Everett RD, Wechsler SL, et al. 2006 Herpes simplex virus type 1 ICP0 localizes in the stromal layer of infected rabbit corneas and resides predominantly in the cytoplasm and/or perinuclear region of rabbit keratocytes. J. Gen. Virol. 87 2817-2825

Nguyen ML and Blaho JA 2007 Apoptosis during herpes simplex virus infection. Adv. Virus Res. $6967-97$

Nguyen ML and Blaho JA 2009 Cellular players in the herpes simplex virus dependent apoptosis balancing act. Viruses $1965-978$

Niederkorn JY, Meyer DR, Ubelaker JE and Martin JH 1990 Ultrastructural and immunohistological characterization of the SIRC corneal cell line. In Vitro Cell. Dev. Biol. 26 923-930

Orosz L, Gallyas É, Kemény L, Mándi Y, Facskó A and Megyeri K 2010 Involvement of p63 in herpes simplex virus-1-induced demise of corneal cells. J. Biomed. Sci. 1747

Orvedahl A, Alexander D, Tallóczy Z, Sun Q, Wei Y, Zhang W, Burns D, Leib DA, et al. 2007 HSV-1 ICP34.5 confers neurovirulence by targeting the Beclin 1 autophagy protein. Cell Host Microbe 1 23-35

Pinazo-Durán MD, Zanón-Moreno V, García-Medina JJ and Gallego-Pinazo R 2013 Evaluation of presumptive biomarkers of oxidative stress, immune response and apoptosis in primary open-angle glaucoma. Curr. Opin. Pharmacol. 13 98-107

Poznic M 2009 Retinoblastoma protein: a central processing unit. $J$. Biosci. 34 305-312

Roizman B and Pellett PE 2001 The family Herpesviridae: a brief introduction; in Fields virology 4th edition (eds) DM Knipe and PM Howley (Philadelphia: Lippincott Williams \& Wilkins) pp 2381-2397

Rowe AM, St Leger AJ, Jeon S, Dhaliwal DK, Knickelbein JE and Hendricks RL 2013 Herpes keratitis. Prog. Retin. Eye Res.32 88-101 
Santana S, Bullido MJ, Recuero M, Valdivieso F and Aldudo J 2012 Herpes simplex virus type I induces an incomplete autophagic response in human neuroblastoma cells. J. Alzheimers Dis. $30815-831$

Shirata N, Kudoh A, Daikoku T, Tatsumi Y, Fujita M, Kiyono T, Sugaya Y, Isomura H, et al. 2005 Activation of teleangiectasiamutated DNA damage checkpoint signal transduction elicited by herpes simplex virus infection. J. Biol. Chem. 280 30336-30341

Silva RA, Berrocal AM, Moshfeghi DM, Blumenkranz MS, Sanislo S and Davis JL 2013 Herpes simplex virus type 2 mediated acute retinal necrosis in a pediatric population: case series and review. Graefes Arch. Clin. Exp. Ophthalmol. 251 559-566

Tallóczy Z, Jiang W, Virgin HW, Leib DA, Scheuner D, Kaufman RJ, Eskelinen EL and Levine B 2001 Regulation of starvationand virus-induced autophagy by the eIF2alpha kinase signaling pathway. Proc. Natl. Acad. Sci. USA 99 190-195

Tallóczy Z, Virgin WH and Levine B 2006 PKR-dependent autophagic degradation of Herpes simplex virus type 1. Autophagy 2 24-29

Tovilovic G, Ristic B, Siljic M, Nikolic V, Kravic-Stevovic T, Dulovic M, Milenkovic M, Knezevic A, et al. 2013 mTORindependent autophagy counteracts apoptosis in herpes simplex virus type 1-infected U251 glioma cells. Microbes Infect. 15 $615-624$
Vemuganti GK, Murthy SI and Das S 2011 Update on pathologic diagnosis of corneal infections and inflammations. Middle East Afr. J. Ophthalmol. 18 277-284

Vemuganti GK, Reddy K, Iftekhar G, Garg P and Sharma S 2004 Keratocyte loss in corneal infection through apoptosis: a histologic study of 59 cases. BMC Ophthalmol. 416

West-Mays JA and Dwivedi DJ 2006 The keratocyte: corneal stromal cell with variable repair phenotypes. Int. J. Biochem. Cell Biol. 38 1625-1631

Wilson SE 1999 Stimulus-specific and cell type-specific cascades: emerging principles relating to control of apoptosis in the eye. Exp. Eye Res. 69 255-266

Wilson SE, Pedroza L, Beuerman R and Hill JM 1997 Herpes simplex virus type-1 infection of corneal epithelial cells induces apoptosis of the underlying keratocytes. Exp. Eye Res. 64 775-779

Zapata G, Racca L, Tau J and Berra A 2012 Topical use of rapamycin in herpetic stromal keratitis. Ocul. Immunol. Inflamm. 20 354-359

Zhang L, Yan Q, Liu JP, Zou LJ, Liu J, Sun S, Deng M, Gong L, et al. 2010 Apoptosis: its functions and control in the ocular lens. Curr. Mol. Med. 10 864-875

Zoukhri D, Fix A, Alroy J and Kublin CL 2008 Mechanisms of murine lacrimal gland repair after experimentally induced inflammation. Invest. Ophthalmol. Vis. Sci. 49 4399-4406

Corresponding editor: GeEta Vemuganti 